\title{
Kordylewski Dust Clouds: Could They Be Cosmic "Superbrains"?
}

\author{
Robert Temple ${ }^{1}$ and Chandra Wickramasinghe $e^{2,3,4}$ \\ 1 The History of Chinese Science and Culture Foundation, Conway Hall, London, UK \\ ${ }^{2}$ Buckingham Centre for Astrobiology, University of Buckingham, UK \\ ${ }^{3}$ Centre for Astrobiology, University of Ruhuna, Matara, SRI LANKA \\ ${ }^{4}$ Institute for the Study of Panspermia and Astrobiology, Gifu, JAPAN \\ Email: ncwick@gmail.com
}

\begin{abstract}
Recent astronomical observations combined with dynamical simulations have led to a possible confirmation of the existence of the much disputed stable dust clouds (Kordylewski Dust Clouds) at the Lagrange libration points of the Earth-Moon system. The new data leads to an estimate of the size of the cloud at L5 as well as of the average radii of the scattering/polarizing dust particles in the cloud's interior. The diameter of the cloud is somewhat less than 3 times the Earth's diameter, and the average grain radius is estimated at $\sim 3 \times 10^{-5} \mathrm{~cm}$, consistent with bacterial-type cells, with a mean separation of less than $1 \mathrm{~cm}$. Such grains, most likely elongated on the average (rod-like bacteria), and photoelectrically charged to a few eV, would acquire a spin through collisions with gas atoms and thus could act as emitters and absorbers of longwave electromagnetic radiation. We speculate that the entire Kordylewski Dust Cloud comprised of such particles has the potential to acquire electromagnetic connectivity with an information storage/processing capacity akin to a form of intelligence.
\end{abstract}

Keywords: Kordylewski dust clouds, interplanetary dust, cosmic biology, extraterrestrial intelligence

\section{Introduction}

The existence of large stable dust clouds at the Lagrange libration points L4 and L5 of the Earth-Moon system appears to have been finally confirmed by a combination of numerical dynamical simulations and polarimetric studies (Sliz-Balogh et al, 2018, 2019). An initial tentative observation of such clouds was reported in 1961 by the Polish astronomer Kazimierz Kordylewski following which they came to be known as Kordylewski Dust Clouds (KDC) (Kordylewski, 1961). The existence of these clouds has, however, been questioned for over 3 decades mainly because of the difficulty of interpreting minute enhancements of night sky brightness in relation to other possible causes. There were also early attempts to detect centimetre to metre-sized bolides in the putative KD clouds using RADAR with negative results and these have been widely considered as disproof of their existence (Roosen and Wolff, 1969; see also, Hou et al, 2015). It is clearly desirable to repeat the earlier RADAR observations and also possibly deploy LiDAR (Light Detection And Ranging) measurements to look for returns from smaller particles to establish their presence.

In two recent publications Sliz-Balogh et al $(2018,2019)$ focussed their attention on the particular dust cloud (KDC) at the L5 point of the Earth-Moon system using sensitive polarimetric techniques. They found clear evidence that a cloud of submicron dust does indeed exist there through examination of polarized scattered light that varied with time (Sliz-Balogh et al, 2019). The cloud appeared to be "dynamic" and contained within it smaller dust clouds, perhaps displaying a cellular-like structure. Although evidence was cited to support the presence ferric and silicate particles we cannot rule out, on the basis of available evidence, the presence of a dominant contribution of carbonaceous or organic grains, as indeed is known to be predominantly present in the interplanetary zodiacal cloud, in cometary dust, as well as the interstellar medium (Hoyle and Wickramasinghe, 2000; Steele et al, 2018).

Further definitive confirmation of the existence of KDC's is clearly desirable and we hope this will be done. It will also be important to unravel the fine structure within clouds, including their internal dynamic properties, but these cannot easily be studied from Earth. Such studies will require investigation by dedicated satellite and astronautical studies at some future date. 
In this article we explore some interesting features of these dust clouds particularly if they are comprised of particles that include a significant biological component.

\section{Inferred Properties of the L5 KDC}

The stability and existence of a KDC at the L5 point has been modelled using 3-D by dynamical simulations, and its actual existence confirmed by means of polarimetric observations of scattered light (Sliz-Balogh et al, 2018,2019). The inferred angular extent of the scattering dust cloud at L5 has been estimated at between $\theta=6$ and 7 degrees. At the known distance of L5, $\mathrm{r}=3.84 \times 10^{9} \mathrm{~cm}$, this angular extent transfers to an average cloud diameter $D$ given by

$$
D \approx \frac{\theta}{360} 2 \pi r \cong 4.35 \times 10^{8} \mathrm{~cm}
$$

This is to be compared with the Earth's diameter of $\sim 1.27 \times 10^{8} \mathrm{~cm}$.

For a spherical particle of radius a (silicate grain or an organic grain typified for example by a bacterium) the cross-section for scattering of sunlight is

$$
C_{s c a} \cong Q_{s c a} \pi a^{2}
$$

with $Q_{s c a}$ having a value close to 1 at optical wavelengths (eg. Wickramasinghe, 1973). For an assembly of such grains in the cloud the average mass scattering coefficient is thus

$$
\kappa_{s c a} \cong \frac{\pi a^{2} Q_{s c a}}{\frac{4}{3} \pi a^{3} s} \approx \frac{3}{4 a s} \mathrm{~cm}^{2} \mathrm{~g}^{-1} \approx 2.5 \times 10^{4} \mathrm{~cm}^{2} \mathrm{~g}^{-1}
$$

assuming $a \sim 3 \times 10^{-5} \mathrm{~cm}, s \sim 1 \mathrm{~g} \mathrm{~cm}^{-3}$.

For significant polarization effects to be observable (Sliz-Balogh et al, 2019) the scattering optical depth through the $4.35 \times 10^{8} \mathrm{~cm}$ path-length of the cloud (equation (1)) must be of order unity, say for instance, $\tau_{s c a} \approx 0.3$. This converts to a mass density of bacterial dust $\rho$ in the cloud given by the equation

$$
0.3 \cong \aleph_{s c a} D \rho \cong 1.09 \times 10^{13} \rho
$$

with the diameter of the cloud given by (1), thus yielding a mass density

$$
\rho \approx 2.75 \times 10^{-14} \mathrm{~g} \mathrm{~cm}^{-3}
$$

The Kordykewski dust cloud at L5 on this basis has a density which is at least $10^{9}$ higher than the density of the ambient interplanetary dust (Allen, 1963). Losses due to the effects of solar radiation, as well as the solar wind effects and small gravitational perturbations which occur mostly in the outermost regions of the KDC will, over long timescales, be made good by the acquisition of new dusty material from comets and the interplanetary medium. The total mass of the cloud observed by virtue of (1) and (5) is thus $\sim 1.17 \times 10^{12} \mathrm{~g}$.

Assuming that a typical dust particle in the cloud has the size characteristics of a bacterial spore with a particle radius a $\sim 3 \times 10^{-5} \mathrm{~cm}$ and mass density $\sim 1 \mathrm{~g} \mathrm{~cm}^{-3}$, we therefore have an average number density of dust particles in the cloud of

$$
\mathrm{n} \approx 2.43 \mathrm{~cm}^{-3}
$$

The mean distance between neighbouring particles is then

$$
\sim \mathrm{n}^{-1 / 3} \sim 0.74 \mathrm{~cm} \text { ! }
$$

very short indeed, and yielding the possibility of inter-particle "communication" if electromagnetic signals can be exchanged. This could be made possible because the bacterial dust would be charged to a potential of a few volts due to the photoelectric effect caused by absorption of solar ultraviolet photons; and collisions with ambient gas would lead to rotation (spinning) at radio frequencies as had been discussed many years ago by (Hoyle and Wickramasinghe, 1970).

\section{$3 \quad$ Emergent Properties of KDC's}

Spinning charged grains, particularly those in the form of elongated needles typified by bacilli, would be efficient absorbers and emitters of electromagnetic radiation. Most interestingly the total number $N$ of such charged dust particles in a KDC (distance of $<1 \mathrm{~cm}$ apart) would be truly vast 


$$
N \approx \frac{\frac{4}{3} \pi R^{3}}{n} \cong 2 \times 10^{26}
$$

With electromagnetic-wave emission/absorption across cloud dimensions as well as electrical connections (charge/current exchanges) between adjacent charged particles just centimetres apart, a $\mathrm{KDC}$, might well be able to function as a gigantic computer/brain capable of storing and processing digital information. We are reminded in the present context of the well-attested cooperative behaviour of bacteria in a wide range of terrestrial settings (Asfahl and Schuster, 2017; Mitchell and Kogure, 2006).

A human brain has only some $10^{11}$ brain cells, and about $10^{15}$ synapses. A KDC (from (8)) may well have a total number of binary connections

$$
\sim{ }^{\mathrm{n}} \mathrm{C}_{2} \approx 10^{52}
$$

between its constituent oscillators, so defining a super-astronomical sum total for its potential computing power. This estimate exceeds the computing power available in all human brains and indeed all other intelligent life on Earth as well by very many orders of magnitude.

Finally, we refer to a few of the remarkable features that are known to characterise dusty complex plasmas and which could also play a role in the present context (eg. Bouchoule, A., 1999; Mikikian et al., 2018). The nucleation and growth of mainly siliceous dust within such plasmas have been documented in several laboratory studies. In our case, however, the dust nucleation process will be side-stepped and condensation within KDC's is likely to occur upon pre-existing interplanetary dust particles which we already argued will probably have a biological component. Thus, we could envisage a population of bacterial particles coated with semi-conducting siliceous mantles that may well have the effect of enhancing inter-particle electronic connectivity. Such speculations may sound far-fetched but they lie within a broad framework of possible outcomes based on known behaviour of complex dusty plasmas.

We might thus be tempted to view the Lagrange Dust Balls as highly structured "intelligent" systems capable of storing and processing "information" and realize that they may have many more surprising and unexpected features. Indeed, such huge stable entities which have presumably endured for astronomical timescales and have steadily grown in complexity over billions of year may display spontaneously evolved phenomena which might resemble those of the most highly complex living entities. This situation is not dissimilar to the brain-like like complexity of the "cosmic web" discussed by Ginsburg et al. (2019), although it is potentially even more impressive in its computational potential. The Dust Balls would contain an intricate combination of charged dusty plasma in gaseous, liquid, and crystal states, with regions of positive charge and regions of negative charge separated from each other by sheaths and double layers, and containing superconducting filaments, so that it would be difficult for us to speak of a Kordylewski Cloud as a whole being singly charged or having a total net charge (or zero net charge), since there would be so many multiple regions of varying charges that a total net charge for each cloud would only be relevant when speaking of the cloud externally, such as in relation to the solar wind which is known to be predominantly positively charged. As a minor point, it should be noted that as silicate particles have been reported in the L5 Kordylewski Cloud, silicate particles within dust tend to be positively charged, a fact first established in 1926 by G. B. Deodar (1927).

It is often said anecdotally that the human brain contains more neurons than there are observable stars in the night sky. But the human brain only fits inside a small skull. A stable dusty complex plasma ball of immense size which has possibly endured for aeons and experienced continual growth and expansion over countless millennia is in principle capable of developing something resembling a much more complex nervous system than a human brain with its average lifetime of $\sim 10^{2}$ years. A complex dust cloud (KDC) which has existed for many millions of years might even have become self-aware.... with all that this implies. It is conceivable that Fred Hoyle's fictional Black Cloud has a reality in the context of KDC's - which of course he could not have recognised in 1957 (Fred Hoyle, 1957).

Acknowledgement. We are grateful to Professor Michael Smith for comments that helped improve an earlier version of this paper.

\section{References}

1. Allen, C.W., 1963. Astrophysical Quantities (London: Althone Press) 
2. Asfahl, K.L., Schuster, M., 2017. FEMS Microbiology Reviews, 41(1), 92

3. Bouchoule, A., 1999. In Dusty Plasmas: Physics, Chemistry and Technological Impacts in Plasma Processing (J. Wiley \& Sons)

4. Deodar, G. B. 1927. Proc. Phys. Soc., 39(3), 243

5. Igenbergs E. et al., 2012. In Levasseur-Regourd A. C. , Hasegawa H., eds, Origin and Evolution of Interplanetary Dust. Kluwer Academic Publish- ers, The Netherlands, p. 45

6. Ginsburg, I. et al., 2019. AdAp, 4(3), 83

7. Hou, X.Y. et al, 2015. Mon.Not.RAS., 454, 4172

8. Hoyle, F., 1957. The Black Cloud (William Heinemann, London.)

9. Hoyle, F. and Wickramasinghe, N.C., 1970. Radio waves from grains in HII regions, Nature, 227, 473

10. Hoyle, F. and Wickramasinghe, N.C., 2000. Astronomical Origins of Life (Kluwer)

11. Kordylewski K., 1961. Acta Astron., 11, 165

12. Mikikian, M., et al, 2018. In Diverse World of Dusty Plasmas - AIP Conference 020019-1

13. Mitchell, J.G. and Kogure, K., 2006. FEMS Microbiology Ecology, 55(1), 3

14. Roosen, R.G. and Wolff, C.L., 1969. Nature, 224, 571

15. Sliz-Balogh, J. et al., 2018. Mon.Not.RAS., 480, 5550

16. Sliz-Balogh, J. et al., 2019. Mon.Not.RAS., in press

17. Steele, E.J. et al, 2018. Prog.Biophys.Mol. Biol., 136, 3-23

18. Wickramasinghe, N.C., 1973. Light Scattering by Small Particles with applications in astronomy (Wiley, NY) 